\title{
Control of a movable robot head using vision-based object tracking
}

\author{
M. Khairudin', G. D. Chen ${ }^{2}$, M. C. Wu ${ }^{3}$, R. Asnawi ${ }^{4}$, Nurkhamid ${ }^{5}$ \\ 1,4,5 Department of Electrical Engineering, Universitas Negeri Yogyakarta, Indonesia \\ ${ }^{2}$ Department of Computer Science and Information Engineering, National Central University, Taiwan \\ ${ }^{3}$ Department of Grad. School of Tech. \& Vocational Edu, National Yunlin University of Science \& Tech., Taiwan
}

\begin{tabular}{l} 
Article Info \\
\hline Article history: \\
Received Jul 31, 2018 \\
Revised Feb 9, 2019 \\
Accepted Mar 10, 2019 \\
\hline
\end{tabular}

\section{Keywords:}

Object tracking

Vision-based

Robot head

\begin{abstract}
This paper presents a visual tracking system to support the movement of the robot head for detecting the existence of objects. Object identification and object position estimation were conducted using image-based processing. The movement of the robot head was in four directions namely to the right, left, top, and bottom of the robot head. Based on the distance of the object, it shifted the object to many points to assess the accuracy of the process of tracking the object. The targeted objects are detected through several processes, namely normalization of RGB images, thresholding, and object marking. The process of tracking the object conducted by the robot head varied in 40 various object points with high accuracy. The further the object's distance to the robot, the smaller the corner of the movement of the robot produced compared to the movement of the robot head to track an object that was closer even though with the same distance stimulant shift object. However, for the distance and the shift of the same object, the level of accuracy showed almost the same results. The results showed the movement of the robot head to track the object under the head of the robot produced the movement with a larger angular error compared to the movement of the robot head in another direction even though with the stimulant distance of the same object position and the distance shift of the same object.
\end{abstract}

Copyright (ㅇ 2019 Institute of Advanced Engineering and Science. All rights reserved.

\section{Corresponding Author:}

M. Khairudin,

Department of Electrical Engineering,

Universitas Negeri Yogyakarta,

No. 1 Colombo Road, Karangmalang, Yogyakarta 55281, Indonesia.

Email: moh_khairudin@uny.ac.id

\section{INTRODUCTION}

The detection and tracking of the object's existence are important and critical especially in automated robot applications so as to interact like human performing autonomous moving and obstacle avoidance [1, 2, 3]. A stimulus-driven data processing and acquisition, active vision sensing coupled with a space-variant sensor, inspired by the visual system of mammalian biology, are essential items [4]. The vision sensors are also applied for machining automation to support intelligence system especially in the robotics system such as humanoid robots [5]. Autonomous motions, especially in an unpredictable area, require several solving in navigation and localisation tasks [6]. Robot vision not allowing direct contact between sensors and objects, such as that in gamma radiation [7], was used as a tool in measuring grasping modules in dark spaces.

Furthermore, to ensure the accuracy of machine vision, calibration of the accuracy and efficiency of the vision sensing system is required [8-9]. The accurate and online calibration is proposed by [10] to ensure the robot can detect the corners from the image. The implementation of vision sensing in color differentiation has been conducted by [11] in identifying Euclidean position of red spheres. Comba, et al., [12] presents the 
vision sensing to develop smart equipment repetitive tasks in an autonomous path. The calibration process on the camera and scanning the robot has to match with the specified grasping point performance [13]. Efforts to make fast responding robots include artificial vision and colony optimization [11]. To interact with the natural surroundings, e.g. in safe workspace monitoring, robots are devised with vision capability [14]. Sahib and Tiziano [15] propose an Ant Colony Optimisation to identify an image in a complex region. Yanling et al [16] apply a vision-based technology to track the seam in real time for robotic gas tungsten arc welding. On the other hand, Montironi [17] applies quality control on production lines through vision-based robots using an adaptive strategy for automatic camera placement. Otherwise, to find the suitable detection for a humanoid robot, Qing Shi et al [18] develops an omnidirectional vision system with five Degrees of Freedom.

Several studies propose various vision-based systems to ensure robot tracking performance, either in single or multi-robots, using various methods, including using a number of cameras [19]. Kinnell [20] simulates a point cloud data into sensor performance to find a fitness of camera positions. Irwansyah et al. [21] implements multiple GigE Vision cameras to support multi-robot tracking based on FPGA. To get a special performance on the vision-based system for robot application, Lu et al [22] propose an efficient deep network to meet its object detection to support efficient and effective optimisation. The purpose of the vision system is that the robot can track the position of the targeted object well [23].

The problem of target detection is one of the most important basic parts of work for robot vision. If the target detection is not successful, the working system in the vision robot will not work. The challenge of this paper is specifically about the accuracy of the camera to detect the position of objects randomly in the form of a red circle. This paper presents the use of vision sensors to direct the robot head to detect the existence of the targeted object. This paper also discusses the comparison among robot head responses and motions after detecting the presence of objects. The tracking results show the motion accuracy that the movement of the robot head with the same object shift stimulus will be more accurate when the webcam vision detects closer object compared to the farther one. However, for the distance and shift of the same object, the accuracy level shows almost the same result.

\section{RESEARCH METHOD}

Figure 1 shows the experimental setup of a part of the robot head used in this work. The rig consists of three main parts: a part of the robot head, a camera sensor, and a processor. The part of the robot head is constructed using a piece of acrylic with specifications as shown in Table 1. The pieces of acrylics are assembled in a shaped head fashion to hold the camera sensor that are actuated by rotors.

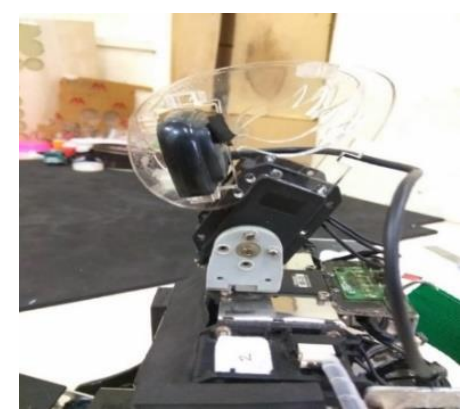

Figure 1. The experimental set up of the vision sensor

Table 1. Parameters of a robot head

\begin{tabular}{ccc}
\hline Parameter & Value & Unit \\
\hline Length & 0.050 & $\mathrm{~m}$ \\
Width & 0.048 & $\mathrm{~m}$ \\
Height of the robot & 0.146 & $\mathrm{~m}$ \\
Servo-motor & 10.0 to 14.8 & volts \\
\hline
\end{tabular}

The sensors used in the system were webcam C170 which was connected to a microcontroller for data processing. The webcam C170 is supported to make a video call and video recording with $640 \times 480$ pixels and 1024 x 768 pixels respectively. The servo motor used in this study was the Dynamixel MX-28 
which was commonly used on humanoid robots with an operating DC voltage of 10 to $14.8 \mathrm{~V}$. The Dynamixel MX-28 servo motor has a high speed for data transmission and includes three pieces of connection namely vcc, data access, and ground. On the other hand, the CM-730 microcontroller was used for data processing in this work.

Data acquisition and control were accomplished by employing a CM-730 microcontroller that provided a direct interface between the processor, actuators, and sensors through signal conditioning circuits. The experimental rig required two-analogue outputs for both motors to cover two degrees of freedom on the robot head movement. The software used includes Visual Studio.net and OpenCV. Figure 2 presents the window shows the webcam scan results. To test the reliability of detection objects, a circle shaped object was used. It was a paper that can be scanned and searched by robots through camera sensors. It is red in color and has a diameter of $0.10 \mathrm{~m}$.

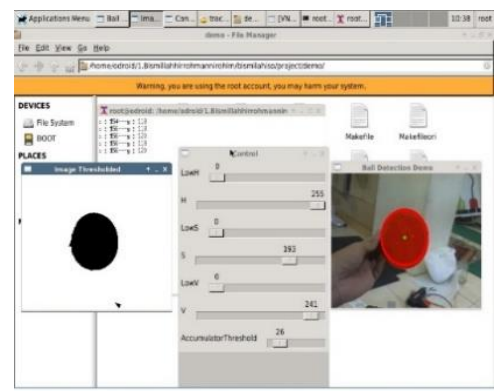

Figure 2. The window for webcam scan

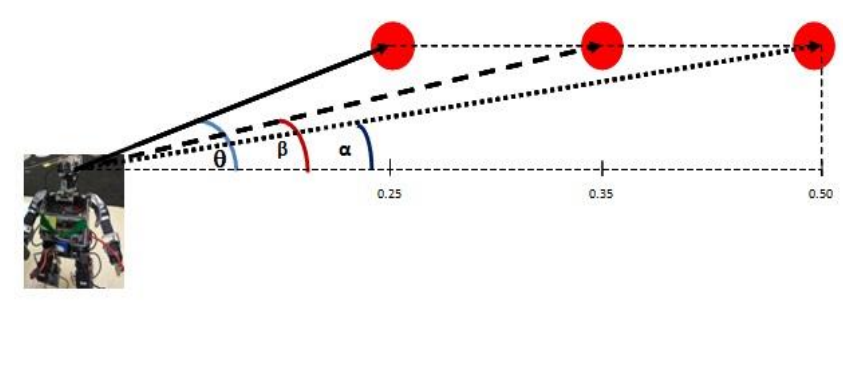

Figure 3. Variations of robot head movements based on object distance and shear distance from the midpoint

Testing the performance of robots in tracking the existence of objects was followed by the movement of the robot head to scan the object according to the position of the detected object. Robot performance testing was also done by dynamically changing the position of the object. This was done in real time to determine the level of reliability of object detection and robot performance in real time. The tests included movement for various directions and the position of the object. Several positions of the object consist of (a) to the right of the robot, (2) to the left of the robot, (3) on the top of the robot, and (4) at the bottom of the robot.

The first test was conducted by placing the red circle paper on the right of the robot head. The distances between the robot head and the detected object were varied. Also with the dynamic distance variable of the detected object to the right in which the center point was the center line of the webcam sensor view. Figure 3 shows various techniques to perform the robot head motion by changing the distance of object shift.

Figure 3 shows that the magnitude of the robot head movement is as in (1)

$$
\alpha<\beta<\theta
$$

where $\alpha, \beta$, and $\theta$ are the motion angles of the robot head for the nearest, medium, and the longest position of the object, respectively. It means when scanning the closest object, the robot will produce a larger angle compared to when the robot head scan the object with a further position

To recognize an object as a target, the robot will scan the red color on the circle object as a reference for tracking the target. Target detection includes processes, namely RGB color normalization, thresholding, and marking objects. In details, the illustration of the process is shown as a flow diagram in Figure 4. The first step in target detection is to normalize each RGB color using (2). The color reference for detecting the target is red based on Figure 2 on the right side. To get the red threshold value in question, the sample is taken, and then to be normalized. The results of red normalization are shown in Table 2. Based on the sampling, it was decided to use a range value of $r=0.5$ to 0.65 , while $g$ is 0.14 to 0.2 .

$$
I_{b i n}(x, y)=\left\{\begin{array}{cc}
0 & I_{B W}(x, y)<T \\
255 & I_{B W}(x, y) \geq T
\end{array}\right.
$$

where $I_{B W}(x, y)$ and $I_{b i n}(x, y)$ were the RGB or grayscale pixels and binary image pixels at $(x, y)$ position respectively, while $T$ was the threshold. 
Next step is the thresholding process. Thresholding is the process of changing the pixel value of an image that meets the threshold value requirements or is in the desired range of values, then to be the desired specified value. The threshold value used in this system is the result of RGB image normalization, namely $r=0.5$ to 0.65 and $g=0.14$ to 0.2 . To speed up the computing process, this study used only $\mathrm{r}$ and $\mathrm{g}$ values because both images can already be used to find the determined red color. The process of changing pixels is performed in two values, 0 and 255 or binarization. This process changes the pixel values in the threshold to 255 , and other values to 0 , thus the image only contains two black (0) and white (255) colors. The binary image results are shown in Figure 2 on the left side.

After the binarization process is completed, the image only consists of two kinds of colors, black and white. The colors of white and black represent the background and the target images respectively. The next step is to mark and to find the target center point. In marking a target, it is based on the size of the white area in the binary image. The target marker is circular and red colored. Examples of target markers are shown in Figure 2 on the right. While the point of target center can be identified using (3).

$$
\begin{aligned}
& x \text { center }=\text { posLeftPixTar }+\left(\frac{\text { posRigthPixTar }- \text { posLeftPixTar }}{2}\right) \\
& y \text { center }=\text { posTopPixTar }+\left(\frac{\text { posBottomPixTar }- \text { posTopPixTar }}{2}\right)
\end{aligned}
$$

where $x$ center and ycenter are the center of row and column, respectively. While posLeftPixTar and posRigthPixTar are the leftmost target line position and the rightmost target row position, respectively. Whereas posTopPixTar and posBottomPixTar are the uppermost target column position and the lowermost target column position, respectively.

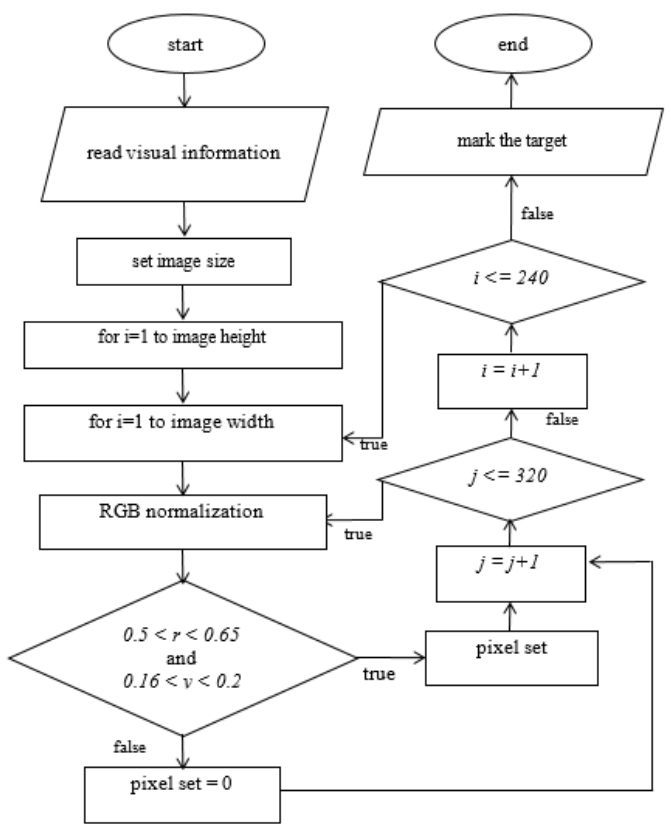

Figure 4. Detecting object process

Table 2. RGB normalisation

\begin{tabular}{ccccccc}
\hline \multirow{2}{*}{ No } & \multicolumn{3}{c}{ Original image } & \multicolumn{3}{c}{ Normalisation image } \\
& $\mathrm{R}$ & $\mathrm{G}$ & $\mathrm{B}$ & $\mathrm{R}$ & $\mathrm{G}$ & $\mathrm{B}$ \\
\hline 1 & 173 & 42 & 55 & 0.645 & 0.154 & 0.203 \\
2 & 128 & 36 & 42 & 0.626 & 0.173 & 0.202 \\
3 & 183 & 48 & 62 & 0.628 & 0.163 & 0.211 \\
4 & 191 & 46 & 64 & 0.638 & 0.152 & 0.212 \\
5 & 212 & 59 & 72 & 0.621 & 0.171 & 0.209 \\
6 & 216 & 53 & 70 & 0.640 & 0.155 & 0.206 \\
7 & 208 & 55 & 70 & 0.628 & 0.164 & 0.210
\end{tabular}




\section{RESULTS AND ANALYSIS}

To assess the target detection and to find out how far the robot can recognize the target, various positions were used. As explained earlier, the introduction of targets based on red objects with RGB color normalization values is in the range of $r=0.5$ to $0.65, g=0.14$ to 0.2 . After the target is identified, then it is marked with a red circle. Tests are carried out by varying distances with the position of the target on the right, left, top and bottom of the robot so that the movement of the head of the robot will follow facing right, left, up and down according to the movement of the target. Tests are carried out in an indoor environment and are regulated in the condition that there is no red other than the target object so that the target detection error can be reduced.

Table 3 presents the results of performance testing when the robot was tracking objects located on the right. Table 3 shows that the more distant the censored object it will cause the movement of the robot head to the direction of changing objects with increasing angle of motion, although it is based on the distance of the same object shift. The angular difference occurred in the robot head movement response to detect the presence of red objects is caused by the difference in distance between the robot head and the object even with the same distance of the object shift. It can be concluded that the farther the object to the robot head will result in a smaller motion angle in the robot head movement than the robot head movement response to the closer object even with the same distance shift stimulus. It can also be concluded that the robot head movement response is more accurate when it detects objects at closer distances compared to distant ones.

Table 3. The performance of the robot head movement in tracking the right object

\begin{tabular}{|c|c|c|c|c|}
\hline \multirow{2}{*}{ No } & \multirow{2}{*}{$\begin{array}{l}\text { Movement of the object to } \\
\text { the right }(\mathrm{m})\end{array}$} & \multicolumn{3}{|c|}{ The robot movement to the right (degree) } \\
\hline & & distance of $0.25 \mathrm{~m}$ & distance of $0.35 \mathrm{~m}$ & distance of $0.50 \mathrm{~m}$ \\
\hline 1 & 0.00 & 0 & 0 & 0 \\
\hline 2 & 0.03 & 7 & 5 & 3 \\
\hline 3 & 0.06 & 14 & 10 & 6 \\
\hline 4 & 0.09 & 20 & 15 & 9 \\
\hline 5 & 0.12 & 26 & 20 & 12 \\
\hline 6 & 0.15 & 35 & 25 & 15 \\
\hline 7 & 0.18 & 42 & 30 & 18 \\
\hline 8 & 0.21 & 50 & 35 & 20 \\
\hline 9 & 0.24 & 55 & 41 & 25 \\
\hline 10 & 0.27 & 62 & 45 & 27 \\
\hline
\end{tabular}

The robot head movement to the right when tracking objects spaced $0.25 \mathrm{~m}$ sustains linear changes with an increasing range of 6 to 9 degrees as a result of changes in object shifts of $0.03 \mathrm{~m}$. The highest change of robot head movement is 9 degrees and this occurs when the object is shifting from $0.12 \mathrm{~m}$ to $0.15 \mathrm{~m}$ from the center point.

Similarly, the angle of the robot head movement to the right on the detection of objects spaced $0.35 \mathrm{~m}$ sustains a linear change with an increasing range of 5 degrees as a result of changes in an object shift each $0.03 \mathrm{~m}$. The highest movement of the robot head moves by 6 degrees when the object sustains a shift from $0.21 \mathrm{~m}$ to $0.24 \mathrm{~m}$. Meanwhile, the change in the movement of the robot's lowest head is 4 degrees.

While the angle of movement of the robot head to the right on the object detection is $0.50 \mathrm{~m}$ with a linear change in an increasing range between 3 degrees to 5 degrees as a result of changes in an object shift of $0.03 \mathrm{~m}$. The highest change in the movement of the robot head of 5 degrees occurs when the object had a shift from $0.21 \mathrm{~m}$ to $0.24 \mathrm{~m}$ from the center. Meanwhile, the lowest change in the movement of the robot head of 2 degrees happens as much as two changes that shift after a distance of $0.18 \mathrm{~m}$ to $0.27 \mathrm{~m}$ from the center.

On the other hand, Table 4 describes the robotic head's response that is almost similar as it occurs when the robot head moves to the right in the opposite direction only. When the robot head is moved to the left of the robot, there is also a difference in the angular response of the robot head movement when it detects the presence of a red object due to the difference in distance between the robot head and the object even with the same distance of the object shift. Table 3 also exposes a relatively similar robot head movement response compared to when tracking the object to the right.

A little different with the movement of the robot head to the right, the angle of the robot head motion to the left on the detection of objects spaced $0.25 \mathrm{~m}$ changes with an increasing range of 5 to 8 degrees as a result of changes in object shift each $0.03 \mathrm{~m}$. The highest change of motion of the robot head moves by 8 degrees when the object sustains a shift from $0.18 \mathrm{~m}$ to $0.21 \mathrm{~m}$ from the center point.

Changes in the movement of the robot's head to the left on the detection of objects spaced $0.35 \mathrm{~m}$ changes with a range of increase of about 4 degrees to 6 degrees as a result of changes in an object shift each $0.03 \mathrm{~m}$. This happens at all object displacement distances. 
While the angle of motion of the head of the robot to the left on the detection object was $0.50 \mathrm{~m}$ which occurs in the range of 2 degrees to 5 degrees. The lowest angle movement occurs when it changes from $0.24 \mathrm{~m}$ to $0.27 \mathrm{~m}$. While the largest movement of the angle occurred when the movement is from $0.21 \mathrm{~m}$ to $0.24 \mathrm{~m}$. While the other relatively stable movement of the angle occurs at about 3 degrees.

Furthermore, Table 5 exposes a change in the angle of the robot head motion upward which appears to be heavier resulting in fewer motion responses than the movement to the right or left and bottom. The change in the angle of the robot head moves upward on the detection of objects spaced $0.25 \mathrm{~m}$ sustains linear changes with an increasing range of 4 to 8 degrees as a result of changes in an object shift each $0.03 \mathrm{~m}$. The smallest angular motion change occurs when the object changed from $0.21 \mathrm{~m}$ to $0.24 \mathrm{~m}$, i.e., with a motion angle of 4 degrees. While the change in motion of the robot head is the highest move of 8 degrees when the object is shifting at some point.

Table 4. Performance of robot head movement in detecting left objects

\begin{tabular}{ccccc}
\hline No & $\begin{array}{c}\text { Movement } \\
\text { of the object } \\
\text { to the left } \\
(\mathrm{m})\end{array}$ & $\begin{array}{c}\text { The robot movement to the left (degree) } \\
\text { distance of } \\
0.25 \mathrm{~m}\end{array}$ & $\begin{array}{c}\text { distance of } \\
0.35 \mathrm{~m}\end{array}$ & $\begin{array}{c}\text { distance of } \\
0.50 \mathrm{~m}\end{array}$ \\
\hline 1 & 0.00 & 0 & 0 & 0 \\
2 & 0.03 & 7 & 5 & 3 \\
3 & 0.06 & 15 & 10 & 7 \\
4 & 0.09 & 21 & 16 & 9 \\
5 & 0.12 & 26 & 20 & 12 \\
6 & 0.15 & 35 & 25 & 15 \\
7 & 0.18 & 42 & 30 & 18 \\
8 & 0.21 & 50 & 35 & 20 \\
9 & 0.24 & 55 & 41 & 25 \\
10 & 0.27 & 63 & 45 & 27 \\
\hline
\end{tabular}

Table 5. Performance of robot head movement in detecting upper objects

\begin{tabular}{ccccc}
\hline No & $\begin{array}{c}\text { Movement } \\
\text { of the object } \\
\text { to upward } \\
(\mathrm{m})\end{array}$ & $\begin{array}{c}\text { Movement of robot up (degree) } \\
\text { distance of } \\
0.25 \mathrm{~m}\end{array}$ & $\begin{array}{c}\text { distance of } \\
0.35 \mathrm{~m}\end{array}$ & $\begin{array}{c}\text { distance of } \\
0.50 \mathrm{~m}\end{array}$ \\
\hline 1 & 0.00 & 0 & 0 & 0 \\
2 & 0.03 & 7 & 5 & 3 \\
3 & 0.06 & 13 & 9 & 5 \\
4 & 0.09 & 19 & 15 & 9 \\
5 & 0.12 & 26 & 20 & 12 \\
6 & 0.15 & 34 & 25 & 15 \\
7 & 0.18 & 42 & 30 & 18 \\
8 & 0.21 & 50 & 35 & 20 \\
9 & 0.24 & 54 & 40 & 24 \\
10 & 0.27 & 62 & 45 & 27 \\
\hline
\end{tabular}

While the angle of the robot head moves upwards on the detection of objects spaced $0.35 \mathrm{~m}$ sustains a relatively stable change with an increasing range of 5 degrees as a result of changes in an object shift each $0.03 \mathrm{~m}$. Except while moving from changing the object from $0.03 \mathrm{~m}$ to $0.06 \mathrm{~m}$, the motion of the robot head moves only 4 degrees.

Meanwhile, the change in the angle of the robot head motion upward on the detection of the object is $0.50 \mathrm{~m}$ undergoing changes almost equal to the previous tables which are about 3 degrees as a result of the change of object shift every $0.03 \mathrm{~m}$. Changes in the motion of the robot head are the smallest that occurs when tracking the movement of objects at some points from the center point, robot head is only able to move 2 degrees.

In other phenomena, Table 6 describes a robotic head's response that is almost similar as it does when the robot head moves upward just in the opposite direction. There is a slight difference with the upward movement of the robot head; the downward angle of the robot head on the detection of the $0.25 \mathrm{~m}$ spaced object with a steady change in the range of 5 to 9 degrees increase as a result of the shifting of each $0.03 \mathrm{~m}$. The highest movement of the robot head by 9 degrees occurs when the object sustains a shift from $0.24 \mathrm{~m}$ to $0.27 \mathrm{~m}$ from the center point. While the smallest response is with a motion of only 5 degrees just when moving the object from $0.21 \mathrm{~m}$ to $0.24 \mathrm{~m}$.

Table 6. Performance of robot head movement in detecting bottom objects

\begin{tabular}{ccccc}
\hline No & $\begin{array}{c}\text { Movement of the object } \\
\text { to the bottom }(\mathrm{m})\end{array}$ & \multicolumn{3}{c}{ Movement to the bottom (degree) } \\
& distance of $0.25 \mathrm{~m}$ & distance of $0.35 \mathrm{~m}$ & distance of $0.50 \mathrm{~m}$ \\
\hline 1 & 0.00 & 0 & 0 & 0 \\
2 & 0.03 & 7 & 5 & 3 \\
3 & 0.06 & 14 & 11 & 7 \\
4 & 0.09 & 20 & 16 & 10 \\
5 & 0.12 & 36 & 20 & 13 \\
6 & 0.15 & 42 & 25 & 15 \\
7 & 0.18 & 50 & 31 & 18 \\
8 & 0.21 & 55 & 35 & 20 \\
9 & 0.24 & 64 & 40 & 25 \\
10 & 0.27 & & 45 & 27 \\
\hline
\end{tabular}


Changes in the movement of the head of the robot downward on the detection of objects spaced 0.35 $\mathrm{m}$ sustains almost stable changes with the range of increase of about 4 to 6 degrees as a result of changes in an object shift each $0.03 \mathrm{~m}$. This happens at all object displacement distances. In contrast to the other direction of motion, the movement of the robot head down on the detection of objects spaced $0.50 \mathrm{~m}$ fluctuates with a range of movement of about 2 to 5 degrees. Fluctuating magnitude of the head angle of the robot downward direction is caused by the force of gravity. Figures 5, 6 and 7 present differences in the angle of movement of the robot head based on the distance of the shift of the same object with the variations of movement to the right, left, top and bottom for the object spacing $0.25 \mathrm{~m}, 0.35 \mathrm{~m}$ and 0.50 respectively as follows.

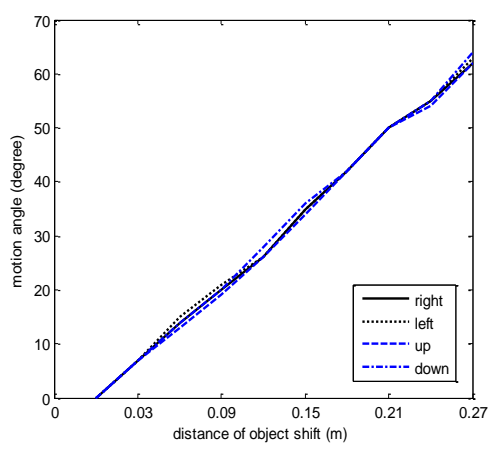

Figure 5. Comparison of response with object distance $0.25 \mathrm{~m}$ from the robot

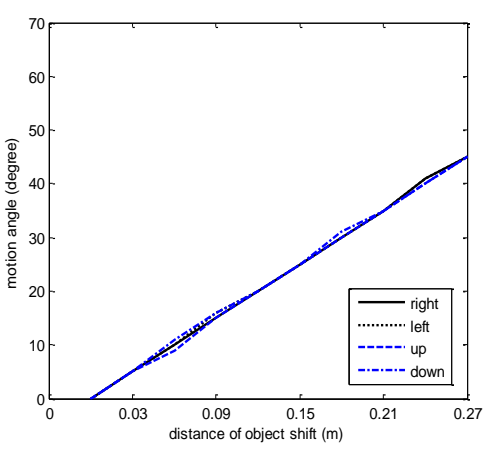

Figure 6. Comparison of response with object distance of $0.35 \mathrm{~m}$ from the robot

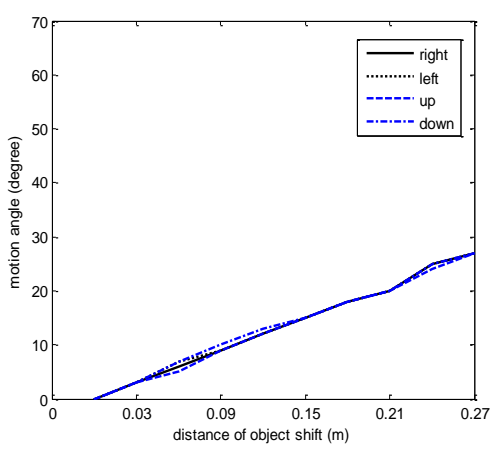

Figure 7. Comparison of response with object distance of $0.50 \mathrm{~m}$ from the robot

It can be seen, the robot movement response for the same object shift distance is that the robot head movement is larger when the object is closer to the robot. It can be concluded that the motion of robot head is the biggest, medium and the smallest degrees for the distance of the object shift of $0.25 \mathrm{~m}, 0.35 \mathrm{~m}$, and 0.50 $\mathrm{m}$, respectively. The results also show for the same object distance, the result of object tracking causes the angle of motion of the robot head at a relatively equal value.

Differences or errors in the direction of the head of the robot when moving in the other direction (e.g. right and left) with the same object distance can be searched by using the Mean Square Error (MSE) formula as shown in the following (4).

$$
M S E=\frac{1}{n} \sum_{i=1}^{n}\left(\theta_{r_{i}}-\theta_{l_{i}}\right)^{2}
$$

where $n, \theta_{r}$ and $\theta_{l}$ are the number of data, motion angle in one side and motion angle in other sides (for example in right and left sides), respectively.

Using (4), MSE can be searched among the directions of motion of the robot head at every distance of the object position. Table 7 shows MSE values among the directions of the robot head movement. Table 7 shows that the MSE changes in the direction of the robot head movements at the object distance of $0.25 \mathrm{~m}$ obtained the smallest MSE values that occur between the movement to the right and to the left that is the value of 0.3 . This is very reasonable because for the movement of the robot head to the right or left with the same power to produce almost the same thrust force so that the two directions are relatively the same direction. Meanwhile, the largest MSE value for object sliding distance in the 0.25 area occurs between up and down movements with a value of 1.5. This highest MSE value can occur because the movement of the robot head down with the same power produces a larger thrust force due to gravity factor. Meanwhile, the average MSE change in the direction of the head of the robot at a distance of $0.25 \mathrm{~m}$ is 0.83 .

While the value on the change of head movement of the robot at the distance of $0.35 \mathrm{~m}$ objects obtained the largest MSE value of 0.6 that occurs between up and down movements, while the smallest MSE of 0.1 occurs between the movement to the right and left. This happens because the movement of the robot head down with the same power produces a larger thrust force so that the resulted angle is greater. As for the average value of MSE change of head movement of the robot at the object distance of $0.35 \mathrm{~m}$ is 0.32 . 


\begin{tabular}{cccc} 
Table 7. MSE value between the directions of robot head movement \\
\cline { 2 - 4 } No & $\begin{array}{c}\text { The Direction of } \\
\text { robot head moves }\end{array}$ & $\begin{array}{c}\text { Distance of } \\
\text { object shift }(\mathrm{m})\end{array}$ & MSE score \\
\hline 1 & Right and Left & 0.25 & 0.3 \\
2 & Right and Left & 0.35 & 0.1 \\
3 & Right and Left & 0.50 & 0.1 \\
4 & Left and Top & 0.25 & 1.1 \\
5 & Left and Top & 0.35 & 0.3 \\
6 & Left and Top & 0.50 & 0.5 \\
7 & Top and Under & 0.25 & 1.5 \\
8 & Top and Under & 0.35 & 0.6 \\
9 & Top and Under & 0.50 & 0.7 \\
10 & Right and Top & 0.25 & 0.4 \\
11 & Right and Top & 0.35 & 0.2 \\
12 & Right and Top & 0.50 & 0.2 \\
13 & Left and Under & 0.25 & 0.8 \\
14 & Left and Under & 0.35 & 0.3 \\
15 & Left and Under & 0.50 & 0.2 \\
16 & Right and Under & 0.25 & 0.9 \\
17 & Right and Under & 0.35 & 0.4 \\
18 & Right and Under & 0.50 & 0.3 \\
\hline
\end{tabular}

Table 7 also describes the MSE changes in the direction of the head of the robot at a distance of 0.50 $\mathrm{m}$ object with the smallest MSE value occurring between the movements to the right and left which is 0.1 . While the largest MSE value occurs between up and down movement of 0.7 and this condition is the same as in the object shift at a distance of $0.25 \mathrm{~m}$ and $0.35 \mathrm{~m}$. While the average value of MSE changes of the motion angle of the robot head at the object distance of $0.50 \mathrm{~m}$ is 0.33 .

Overall the largest MSE value is 1.5 that occurs between upward and downward movements at the point of movement of the robot head with a distance of $0.25 \mathrm{~m}$. The same phenomenon is shown at the object distance of 0.35 and $0.50 \mathrm{~m}$, that there is a large MSE value when the comparison is done between the upward motion of the robot head with downward movement when tracking the object's existence. This happens because of the influence of gravity, i.e. when the robot is tracking the existence of the object downwards with the same power, it produces more thrust force.

In opposite conditions, the smallest MSE values occur between right to left motions with a value of 0.1 at a distance of $0.35 \mathrm{~m}$. The same phenomenon is shown at the distance of the object 0.25 and $0.50 \mathrm{~m}$, that there is a small MSE value when a comparison is done between the movement of the robot head to the right with the movement to the left when tracking the existence of the object. This happens because of the influence of gravitational force, that is, when the robot is tracking the existence of the object to the right and left with the same power, it produces the same thrust force that produces a relatively similar thrust.

\section{CONCLUSION}

This paper presented a vision-based object tracking system with 2 degrees of freedom movable robot head. The vision-based object tracking performance on the robot head was tested using four directions of robotic movement covering the right, top, left, and bottom. The robot head was used as the center of object movement. The results showed the robot head can accurately track the existence of objects. The closer the object was to the robot, the greater the angle of the robot head moves in comparison with the movement of the robot head to track the object further away even with the same distance-shifting object stimulant. However, for the distance and the shift of the same object, the accuracy level showed almost the same result. A large MSE value occurred between upward and downward movement in the robot head movement angle with at all distances of the object. Medium-small happened between right to left movement at all distance of the object.

\section{ACKNOWLEDGEMENT}

The work was supported by the Ministry of Research, Technology and Higher Education, Republic of Indonesia under the grant of LPPM Universitas Negeri Yogyakarta. 


\section{REFERENCES}

[1] J. R. Terven, et al., "Head-Gestures Mirroring Detection in Dyadic Social Interactions With Computer VisionBased Wearable Devices," Neurocomputing, vol. 175, pp. 866-876, 2016.

[2] A. Gepperth, et al., "The Contribution of Context Information: A Case Study of Object Recognition in an Intelligent Car," Neurocomputing, vol. 94, pp. 77-86, 2012.

[3] C. Caraffi, et al., "A System for Real-Time Detection and Tracking of Vehicles From A Single Car-Mounted Camera," IEEE Intelligent Transportation Systems Conference, pp. 975-982, 2012.

[4] C. Bartolozzi, et al., "eMorph: towards Neuromorphic Robotic Vision," The European Future Technologies Conference and Exhibition, Procedia Computer Science, vol. 7, pp.163-165, 2011.

[5] D. Yongtae, "Intelligent Worm Sorting Using Robot Vision," International Symposium on Robotics and Intelligent Sensors (IRIS 2012), Procedia Engineering, vol. 41, pp. 917-922, 2012.

[6] G. Nikolai and L. Alexander, "Computer Vision for Mobile on Ground Robotics," 25th DAAAM International Symposium on Intelligent Manufacturing and Automation, 2014. DAAAM 2014. Procedia Engineering, vol. 100, pp. 1376-1380, 2015.

[7] T. Maruyama, et al., "Robot Vision System R\&D for ITER Blanket Remote-Handling System," Fusion Engineering and Design Des, 2014.

[8] M. Khairudin, et al., "System Identification and LMI Based Robust PID Control of a Two-Link Flexible Manipulator," TELKOMNIKA Telecommunication Computing Electronics and Control, vol/issue: 12(4), pp. 829-838, 2014.

[9] D. M. Abrham and M. A. Dagnachew, "Soil Characterization and Classification: A Hybrid Approach of Computer Vision and Sensor Network," International Journal of Electrical and Computer Engineering (IJECE), vol/issue: 8(2), pp. 989-995, 2018.

[10] D. Guanglong and Z. Ping, "Online Robot Calibration Based on Vision Measurement," Robotics and ComputerIntegrated Manufacturing, vol. 29, pp. 484-492, 2013

[11] F. G. Luna and A. M. Diaz, "Towards an Artificial Vision-Robotic System for Tomato Identification," IFACPapers OnLine, vol/issue: 49(16), pp. 365-370, 2016.

[12] L. Comba, et al., "Robotics and Automation for Crop Management: Trends and Perspective," Proceeding of International Conference Ragusa SHWA, pp. 471-478, 2010.

[13] E. Misimi, et al., "GRIBBOT-Robotic 3D Vision-Guided Harvesting of Chicken Fillets," Computers and Electronics in Agriculture, vol. 121, pp. 84-100, 2016.

[14] H. Herrero, et al., "Skill Based Robot Programming: Assembly, Vision and Workspace Monitoring Skill Interaction," Neurocomputing, 2016.

[15] K. Sahib and B. Tiziano, "Ant Colony Optimization (ACO) based Data Hiding in Image Complex Region," International Journal of Electrical and Computer Engineering (IJECE), vol/issue: 8(1), pp. 379-389, 2018.

[16] Y. Xu, et al., "Computer Vision Technology for Seam Tracking in Robotic GTAW and GMAW," Robotics and Computer-Integrated Manufacturing, vol. 32, pp. 25-36, 2015.

[17] M. A. Montironi, et al., "Adaptive Autonomous Positioning of A Robot Vision System: Application," Robotics and Computer-Integrated Manufacturing, vol. 30, pp. 489-498, 2014.

[18] Q. Shi, et al., "Design and Implementation of Anomnidirectional Vision System for Robot Perception," Mechatronics, vol. 41, pp. 58-66, 2017.

[19] A. Tanoto, et al., "A Teleoperated Plat-Form for Experiments in Multi-Robotics," Web-Based Control and Robotics Education Springer Verlag, vol. 38, pp. 287-316, 2009.

[20] P. Kinnell, et al., "Autonomous Metrology for Robot Mounted 3D Vision Systems," CIRP Annals-Manufacturing Technology, 2017. http://dx.doi.org/10.1016/j.cirp.2017.04.069.

[21] A. Irwansyah, et al., "FPGA-based Multi-Robot Tracking," Journal of Parallel Distributed Computing, 2017. http://dx.doi.org/10.1016/j.jpdc.2017.03.008.

[22] K. Lu, et al., "Efficient Deep Network for Vision-Based Object Detection in Robotic Applications," Neurocomputing, 2017. doi:10.1016/j.neucom.2017.03.050.

[23] L. Perez, et al., "Robot Guidance Using Machine Vision Techniques in Industrial Environments: A Comparative Review," Sensors, vol/issue: 16(3), pp. 335, 2016.

\section{BIOGRAPHIES OF AUTHORS}

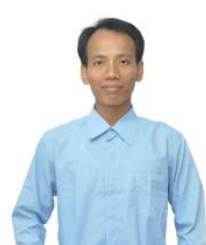

Moh. Khairudin received his bachelor from Universitas Negeri Yogyakarta at the department of electrical engineering in 2002. He graduated from Institute Teknologi Sepuluh Nopember (ITS) with M.Eng. in control electrical engineering in the year of 2004 and completed his $\mathrm{PhD}$ in control electrical engineering in the year of 2012, from Universiti Teknologi Malaysia, Johor, Malaysia. His research interested in the area of Flexible Manipulator, Robotics and Automation, Robust Control and Intelligent Control System. 


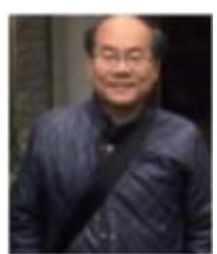

Gwo-dong Chen received his bachelor from Department of Electrical Engineering, National Taiwan University in 1979. He received MS and PhD degrees from Department of Electrical Engineering, National Taiwan University in the years of 1982 and 1991 respectively. Currently he is the CSIE chair professor of Department of Computer Science and Information Engineering, National Central University. His research topics are on Human Computer Interaction especially for technology enhanced learning.

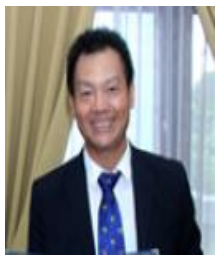

Mingchang Wu received his Ph.D from Purdue University. He is currently a full professor and the dean in National Yunlin University of Science and Technology, Taiwan. He is dedicated to academic research in the field of Vocational education development, Reasoning Skills, and Collaborative projects in educational settings. He has presented papers at several conferences around the world and published in international journal including in SSCI, Scopus, and EI Journals.

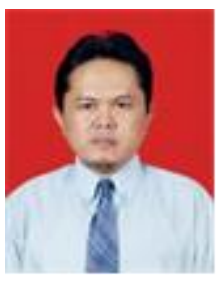

Rustam Asnawi received his bachelor and Master degrees from Department of Electrical Engineering, Universitas Gadjah Mada in 1995 and 2004 respectively. He received PhD degrees from Department of Computer \& Information Sciences, Universiti Teknologi PETRONAS, Malaysia in 2012. His research topics are in Robust synchronization models for Presentation System, Applying a Framework for General Live Multimedia, Multimodal e-Slide Presentation

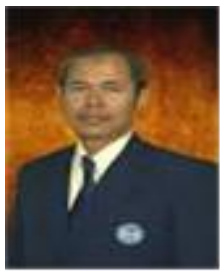

Nurkhamid graduated from Universitas Gadjah Mada (UGM) with BSc. in Electronics and Instrumentation in 1983. He received her MSc. in Computer Science in the year 2004 from UGM and his $\mathrm{PhD}$ in Computer Science and Information Engineering in the year 2007, from National Central University, Zhong-Li, Taiwan. His interested in Building Digital Playgrounds and Educational Games and Virtual Reality/Augmented Reality. 